\title{
Frequency Optimization in FAMPLC Technique for Point to Multipoint Control and Monitoring of Remote Applications
}

\author{
A. Nazem ${ }^{1 *}$ and M. R. Arshad ${ }^{2}$ \\ 1,2 2Underwater Robotics Research Group (URRG), School of Electrical and Electronic Engineering, Universiti Sains \\ Malaysia, Nibong Tebal, Pulau Pinang, Malaysia; alireza_nazem@fltzlogic.com', rizal@eng.usm.my²
}

\begin{abstract}
Background/Objectives: In this paper a novel approach using the FAMPLC technique is presented as a solution for point to multipoint control and monitoring. A mathematical method for frequency optimization is also discussed.

Methods/Statistical Analysis: A dedicated DC to AC inverter as a large signal integrated gateway for power and data in one side and multiple nodes each containing an active resistance as a dummy load on the other sides are considered to establish a distant bidirectional communication platform. A method in which the master unit is programmed to control and monitor the multipoint is discussed.

Results: A mathematical approach for determination of the required frequency and the pulse width ranges are also offered. A compensative calibration sequence and a recommended communicating protocol are also presented.

Conclusion/Application: The herewith presented model has been successfully developed and installed in the campus using 8 nodes in order to control and monitor the fire alarm system.
\end{abstract}

Keywords: FAMPLC, Large Signal Power Line Communication, Frequency Modulated Power Supply, Point to Multipoint Communication.

\section{Introduction}

Huge numbers of applications in the industry require remote access and control. Safety and inaccessibly are the two major demanding reasons. A remote access can be established either on air or using wire [1]. The wireless solutions, although requiring less installation effort, have an inherent drawback due to a couple of reasons. The restricted free band and the line of sight feature [2]. These reasons sound sufficient for discouraging consideration, especially in high-risk applications such as oil and gas industry sectors. The Supervisory Control And Data Acquisition (SCADA) system is commonly considered as a peripheral I/O handler device for Programmable Logic Controllers (PLC) [3]. Apart from any protocol that may be used for interconnection, a SCADA module has to be powered somehow. Preparation of a localized power supply to empower the SCADA system is a crucial procedure in an inaccessible area. In addition, data transmission and cabling efforts are not encouraged for distances beyond a few hundred meters [4]. Finally, the conventional Power Line Communication (PLC) systems, apart from any existing technology, have a limited communication distance due to the practice of Small Signal Superimposed Technique (SSST). SSST is fairly suppressed along the PLC transmission line and more particularly if a Shunt Active Power Filter is applied [5].

Frequency and Amplitude combined Modulations in Power Line Communication (FAMPLC) is introduced as

*Corresponding author:

A. Nazem (alireza_nazem@fltzlogic.com) 
a non-superimposing method in which the power-line is utilized as a communication platform. It is known as a large signal power line communication method which uses parametric values such as Voltage, Current, frequency and the duty cycle [6]. A dedicated DC to AC inverter is designed as the power and data gateway for this purpose which is called the master (or server) module [7]. This technique is a stand-alone embedded system containing a square wave Frequency Modulated Power Supply (FMPS) and Thevenin Equivalent Converter (TEC) circuitries. Figure 1 shows the architecture of the master module in this research.

FMPS is controlled using a Microcontroller circuitry for variable frequencies and duty cycles based on predefined commands. The receiver node as a communicating point is to extract the means of commanding data by analysing the power pulse widths. The transmission procedure from the receiver node to the master is then performed by use of an active resistance as a dummy load. Figure 2 is an illustration of a receiver node's proposed architecture in this research. A successful development of this technique in a Remotely Operated Vehicle (ROV) application is discussed in [8].

\section{Approach and Methods}

Figure 3 illustrates the point to multi-point network model of this research. Each node is considered to be able to handle multiple General Purpose Input Output (GPIO) ports.

The outcome of both FM and AM transmissions are concluded in two parameters:

1- Transmission frequency " $F$ ": in which the periodic term of the received frequency is being analyzed.

2- Transmission duty cycle " $D$ ": in which the mark (or space) term of the frequency is being analyzed.

In fact, according to Figure 1 and 2, a counting process is performed by CPU2. In order to measure the receiving

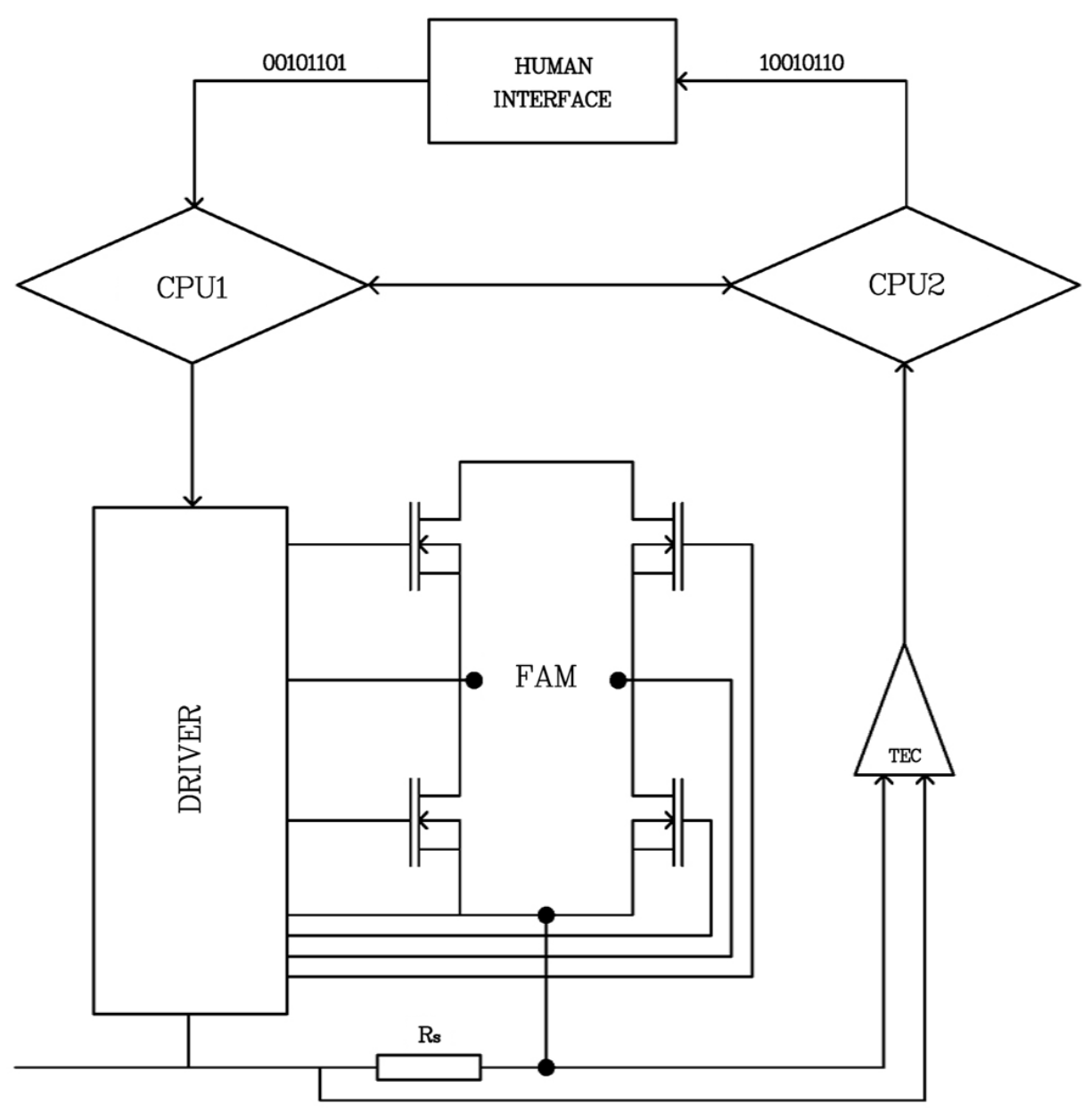

Figure 1. The architecture of a master (server) module. 


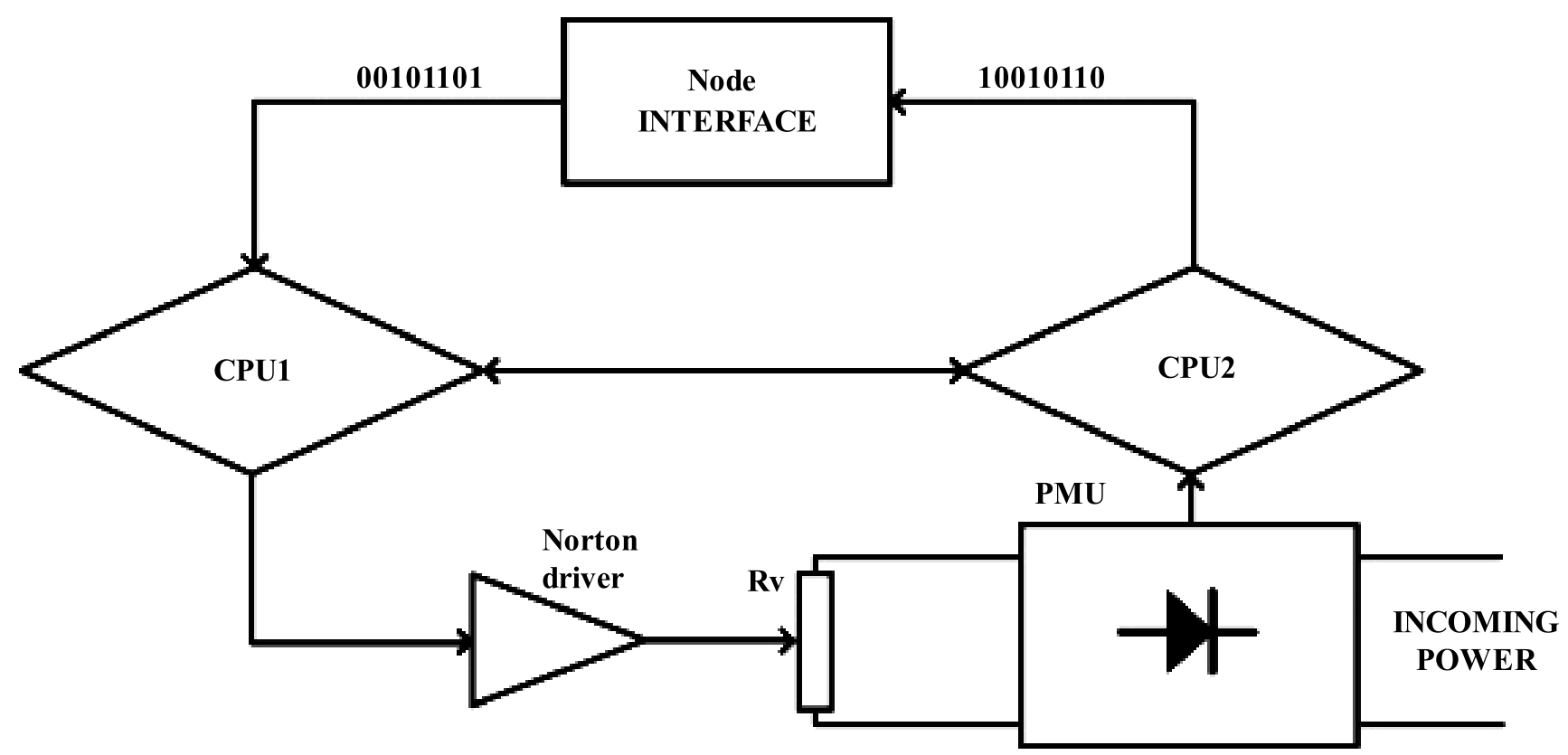

Figure 2. The architecture of a receiver node.

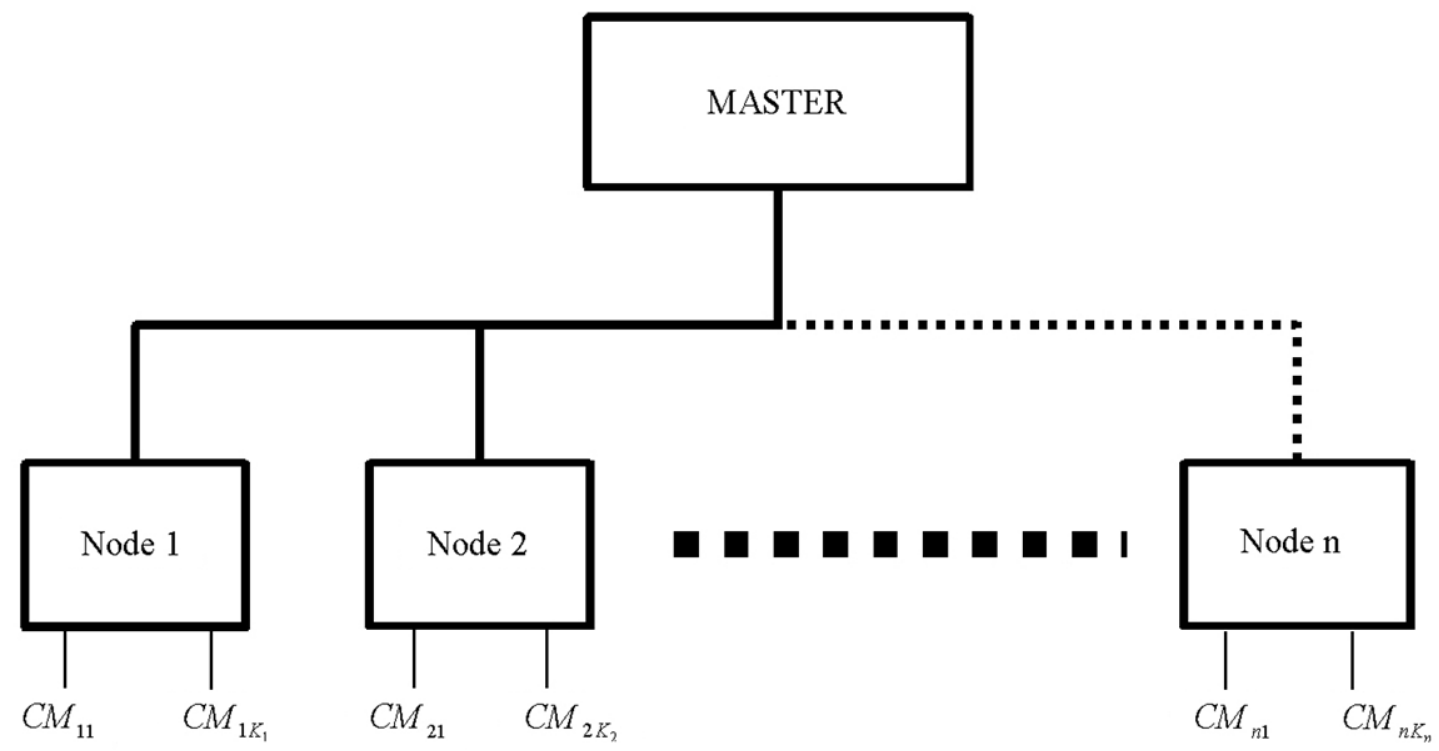

Figure 3. The network model.

frequency, an embedded counter with a specific clock frequency is initiated at the time of every rising (or falling) edge and stops at the next consequent rising (or falling). While, the duty cycle measurement is more commonly a counting process from every rising edge to the consequent falling, thus called the mark measurement process. Moreover, one may desire to measure the space value instead. The processor units used in this research are chosen from the mid-range microcontroller architecture [9]. As an example of this, let's consider a specific command at $F_{1}=40 \mathrm{~Hz}$. Depending on the clock frequency of the CPU a pre-scaled timer is set up. For $F_{C}=20 \mathrm{MHz}$ as the CPU clock, the timer increments are every $t_{C}=2 \mu s$. Thereby, between two rising edges, $C_{P}=12500$ pulses is counted for the period of $T_{1}=12.5 \mathrm{~ms}$. The counter value is the most critical element for both the control and monitoring process in this technique. Each control command and each informative 
data are the pre-stored values in the memory as one or more set of numbers. Meaning, in order to achieve an errorless pulse width count, instead of a solid number, a domain is defined using expression 1 :

$$
C_{P}+\gamma>C_{P}>C_{P}-\gamma
$$

where $\gamma$ is the propagation constant that is included in the program routine. $\gamma$ is to cover the distortion interval of the receiving pulse in order to overcome the controversial errors. Figure 4 is a conceptual illustration for better understanding of the $C_{p}$ distribution in a predefined lookup table. $g_{C}$ is called the protective gap counts and is defined to prevent overlaps. Since the system has to be designed independent from the transmission line, $\gamma$ is set as:

$$
\gamma=100 / t_{C}
$$

In this case, for $F_{C}=20 \mathrm{MHz} \mathrm{CPU}$ clock, $\gamma=50$ propagated counts will be considered and therefore, in the herewith given example, $40.16 \mathrm{~Hz}>F_{1}>39.84 \mathrm{~Hz}$. In order to create a generic expression for calculating the lower and higher frequency limits, let's consider a 16 bits timer module which uses increments every $2 \mu \mathrm{sec}$. It yields to the maximum of $2^{16}=65536$ counts before the counter overflows. Therefore, the maximum pulse width will be $2^{16} \times 2 \mu$ sec. $=0.13 \mathrm{~s}$. The result is a minimum frequency of $7.63 \mathrm{~Hz}$. However, due to the necessity of three major factors:

1- Calibration sequence

$2-$ " $\gamma$ "

$3-"$ " $g_{C}$ "

The minimum frequency is maximized proportionally to:

1- " $C M_{i j}$ " (the number of commands)

2- " $n$ " (the number of slave nodes)

3- Counter (Timer) bits

4- " $F_{C}$ ” (Counter (Timer) frequency).

According to Figure 3 , the $\mathrm{n}^{\text {th }}$ slave node requires the number of $C M_{i j}$ commands. Therefore, the total commands that require a specific pulse width allocation can be obtained from equation 3 :

$$
C M_{T}=\sum_{i=1}^{n} \sum_{j=1}^{K_{n}} C M_{i j}
$$

where $K_{n}$ is the number of required commands (to access available I/Os) in the $\mathrm{n}^{\text {th }}$ node. Taking Figure 4 as a symolic example, the parametric values are as below:

$-g_{C}=\gamma=4$

- $C_{p 1}=9, C_{p 2}=22, C_{p 3}=35$
For ease of understanding, assuming a $2 \mathrm{msec}$ incremental timer yields:

$-26 m s \geq M_{1} \geq 10 m s$

$-52 m s \geq M_{2} \geq 36 m s$

- $62 m s \geq M_{3} \geq 46 m s$

where, $M_{X}$ represents the Mark of the command period $T_{X}$. Figure 5 draws this to picture.

Concluding what has been discussed so far leads us to create the generic expression 4, for the purpose of calculating the required boundaries of a pulse width in an uncategorized frequency model.

$$
C_{P}=g_{c}+\left(1+2 \gamma+g_{c}\right) C M_{T}
$$

Assuming $M_{p}=C_{p} t_{c}$ as the maximum required pulse width, results in:

$$
M_{P}=t_{C}\left[g_{c}+\left(1+2 \gamma+g_{c}\right) \sum_{i=1}^{n} \sum_{j=1}^{K_{n}} C M_{i j}\right]
$$

For example, to be more realistic, in the network of Figure 3, if:

$-\mathrm{n}=10$

- $K_{n}=\left[\begin{array}{lllllllll}4 & 2 & 2 & 4 & 3 & 8 & 6 & 2 & 4\end{array}\right]$ (total of 39 GPIOs)

$-g_{C}=100$

$-\gamma=50$

- $t_{C}=2 \mu$ s (typical value for CPU clock @ 20MHz)

Then, $M_{P}=15.878 \mathrm{~ms}$ will be the maximum pulse width (mark) required for an errorless transmission. In an uncategorized frequency model where duty cycle plays the main role, if $S_{P}=10 \% M_{P}$ (where $S_{P}$ represents the "space" of the pulse), then: $T_{P}=17.4658 \mathrm{~ms} \rightarrow F_{P}=57.25 \mathrm{~Hz}$ will be the minimum required frequency. $T_{P}$ represents the full cycle period and $F_{P}$ specifies the pulse train frequency. The first command pulse may result in a very narrow pulse width. For instance, in the herewith given example since 39 commands are required, $M_{P}=407 \mu$ s will be the pulse width for the first command. Similar to the FM mode, expression 6 is used in current modulation of the AM mode. In this case, $C M_{T}$ is substituted by $d_{n}$ as the $\mathrm{n}^{\text {th }}$ informative data means.

$$
C_{P}=g_{c}\left(d_{n}+1\right)+d_{n}+2 \gamma d_{n}
$$

In FAMPLC technique since the transmission speed versus the transmission distance, wider pulse width however affecting the speed, may result in a lesser count loss due to the edge distortion. To overcome this issue, for a point to multi-point model in which the numbers of commands are 


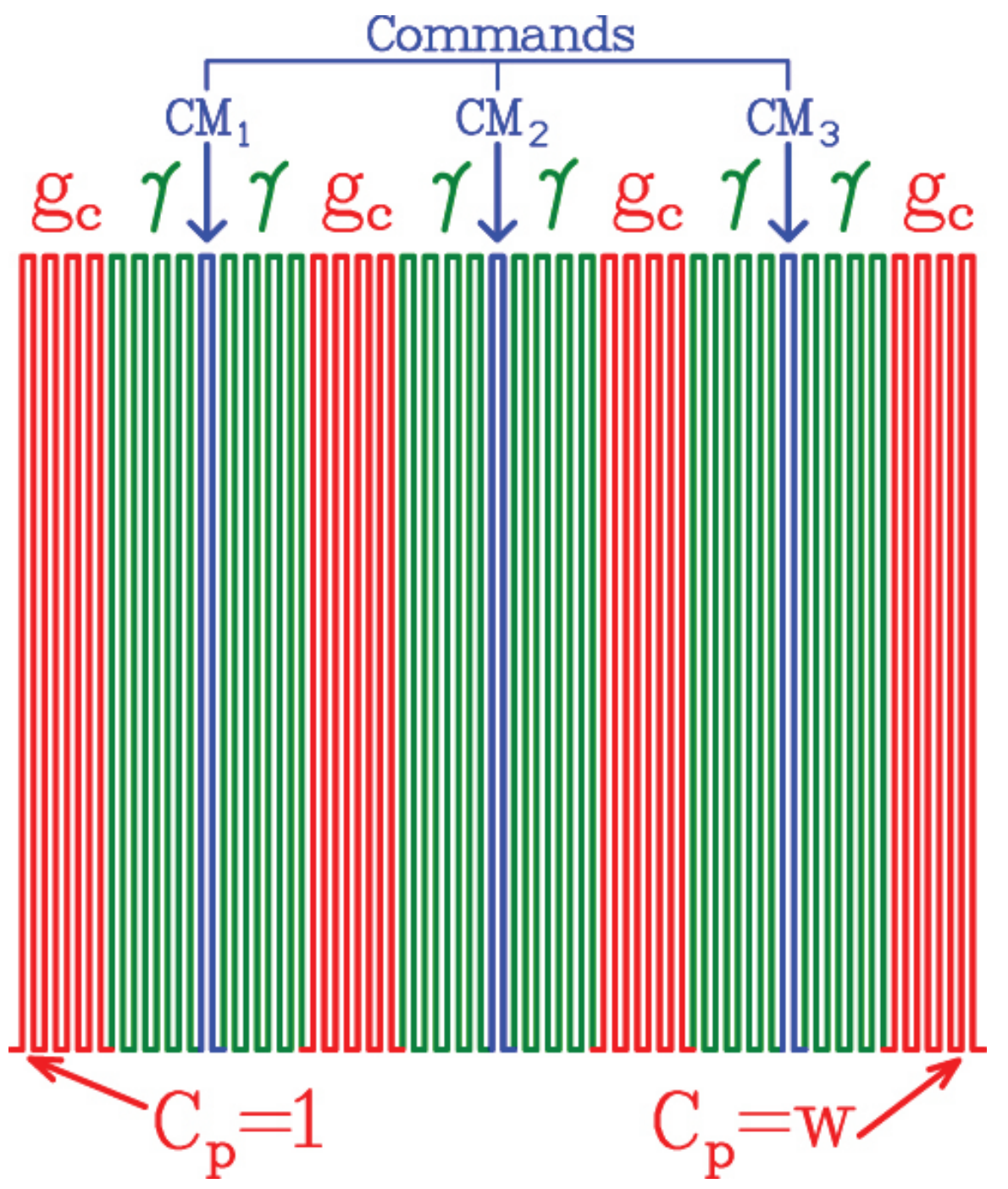

Figure 4. The $\mathrm{C}_{\mathrm{p}}$ distribution in the lookup table.
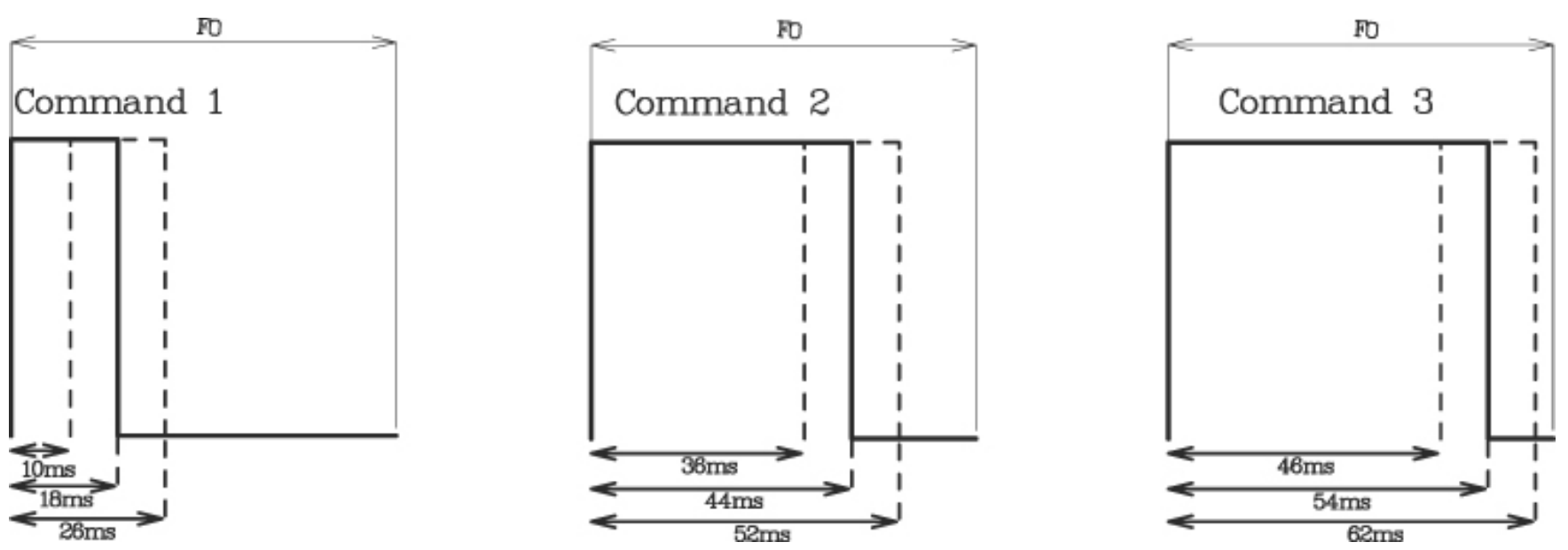

Figure 5. Example for three different Pulse Width Modulations. 
considerably large, categorizing dedicated frequencies will be an effective solution. Let's consider $F_{n}$ as assigned for the $\mathrm{n}^{\text {th }}$ node. Therefore, equation 7 is a simplified equation as a solution.

$$
F_{n} \mid M_{P}=t_{C}\left[g_{c}+\left(1+2 \gamma+g_{c}\right) \sum_{j=1}^{K_{n}} C M_{j}\right]
$$

Meaning that, an equal pulse width may be assigned for different frequencies. An example of this for three imaginary remote devices is tabulated in Table 1.

Table 1. An example of frequency categorization model

\begin{tabular}{llll}
\hline Device & $\begin{array}{l}\text { Dedicated } \\
\text { frequency }\end{array}$ & Pulse width (duty cycle) & Status \\
\hline A & $\mathrm{F}=30 \mathrm{~Hz}$ & $\mathrm{D}=10 \%\left(T_{P}=3.33 \mathrm{~ms}\right)$ & On \\
& & $\mathrm{D}=20 \%\left(T_{P}=6.66 \mathrm{~ms}\right)$ & Off \\
B & $\mathrm{F}=35 \mathrm{~Hz}$ & $\mathrm{D}=11.7 \%\left(T_{P}=3.33 \mathrm{~ms}\right)$ & On \\
& & $\mathrm{D}=23.3 \%\left(T_{P}=6.66 \mathrm{~ms}\right)$ & Off \\
C & $\mathrm{F}=45 \mathrm{~Hz}$ & $\mathrm{D}=15 \%\left(T_{P}=3.33 \mathrm{~ms}\right)$ & On \\
& & $\mathrm{D}=30 \%\left(T_{P}=6.66 \mathrm{~ms}\right)$ & Off \\
\hline
\end{tabular}

\section{Results and Discussion}

For proof of the concepts, the technique presented in this paper has been successfully installed in the campus for the centralization of the fire alarm system. There are eight nodes configured as in figure 6 to communicate with a master unit at the main building. Note that the uncategorized model has been considered for this implementation.

The master unit scans the nodes periodically to check the inputs seeking faulty data. In addition, a localized panic alarm is being activated by each node in an occasion of fault with a command from the master. Figure 7 shows the conceptual picture.

Therefore, one input and one output for each node is considered. To comply with the building standards, an armed cable had to be used. Thus, an additional capacitive distortion due to the shield effect had to be considered. Nevertheless, the power pulse width readings in the FM mode as shown in table 2 were in the expected range.

However, narrow pulse widths in the AM mode somehow fell out of range as shown in table 3.

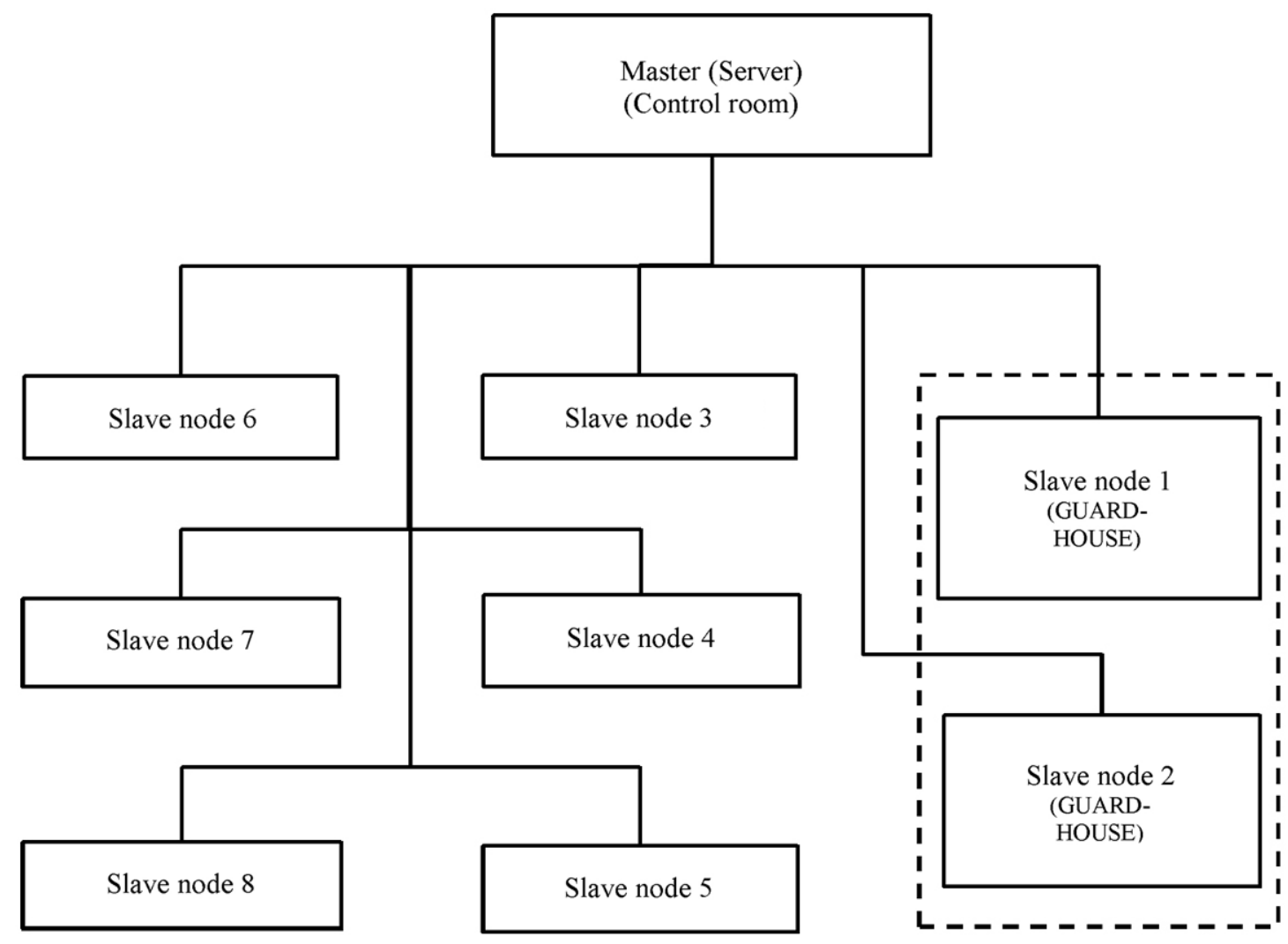

Figure 6. The experimental configuration. 


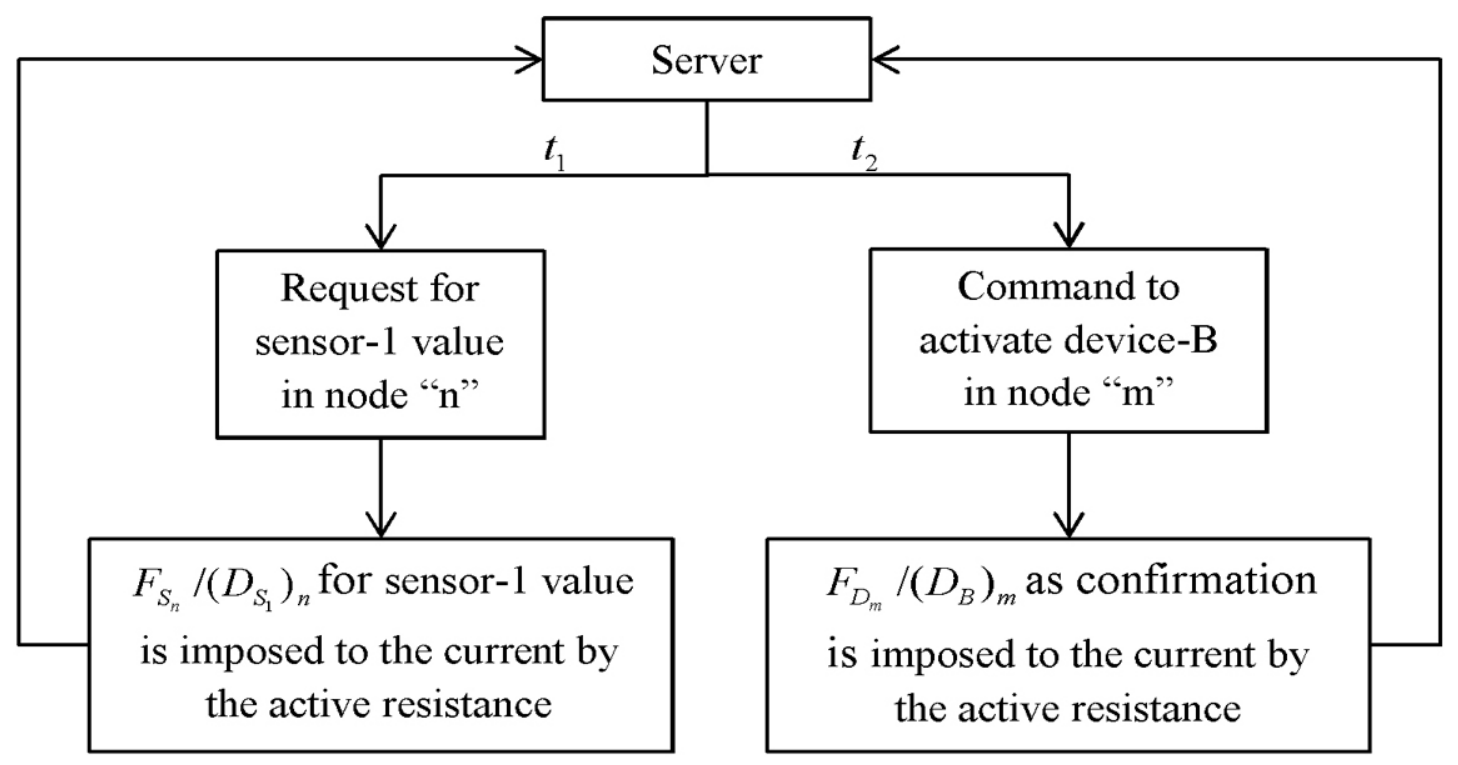

Figure 7. The communication sequence.

Table 2. FM power pulse width counting results by the nodes

\begin{tabular}{lcc}
\hline$C M_{i j}$ & $C_{p}(D \%)($ Sent $)$ & $C_{p}(D \%)$ (Received) \\
\hline$C M_{11}$ & 2366 & 2361 \\
$C M_{12}$ & 2215 & 2214 \\
$C M_{21}$ & 2064 & 2060 \\
$C M_{22}$ & 1913 & 1909 \\
$C M_{31}$ & 1762 & 1759 \\
$C M_{32}$ & 1611 & 1608 \\
$C M_{41}$ & 1460 & 1455 \\
$C M_{42}$ & 1309 & 1307 \\
$C M_{51}$ & 1158 & 1152 \\
$C M_{52}$ & 1007 & 1000 \\
$C M_{61}$ & 856 & 850 \\
$C M_{62}$ & 705 & 701 \\
$C M_{71}$ & 554 & 547 \\
$C M_{72}$ & 403 & 398 \\
$C M_{81}$ & 252 & 246 \\
$C M_{82}$ & 101 & 95 \\
\hline
\end{tabular}

Although considering the $\gamma$ and $g_{C}$ results, there was an overcoming achievement to minimize errors. However, there might be longer damping distortions depending on various characteristics of the transmission line or very long distant remote applications that require thousands of meters of cable. To address all other possible errors, a calibration sequence is introduced.
Table 3. AM current pulse width counting results by server

\begin{tabular}{lcc}
\hline$C M_{i j}$ & $C_{P}(D \%)($ Sent $)$ & $C_{P}(D \%)($ Received $)$ \\
\hline$d_{1}$ & 1158 & 1138 \\
$d_{2}$ & 1007 & 988 \\
$d_{3}$ & 856 & 834 \\
$d_{4}$ & 705 & 679 \\
$d_{5}$ & 554 & 529 \\
$d_{6}$ & 403 & 375 \\
$d_{7}$ & 252 & 201 \\
$d_{8}$ & 101 & 48 \\
\hline
\end{tabular}

\subsection{Calibration}

The so far discussed technique using FAMPLC is designed as an adaptive behavioural transformation to revise and compensate the count losses in crucial conditions [10]. A calibration pulse with a constant value is taught to both server and node modules by the embedded programming. This is meant for internal comparisons to evaluate the amount of pulse width reductions due to any filtering process. For instance, a definite power pulse with parametric values of:

$$
\left\{\begin{array}{l}
F_{c a l}=30 \mathrm{~Hz} \\
D_{c a l}=30 \%
\end{array} \Rightarrow T_{\text {cal }}(\text { mark })=10 \mathrm{~ms}\right.
$$

accompanies every commander pulse-width in advance. With an expectation of $C_{P}=5000, \mathrm{CPU} 2$ in Figure 1 


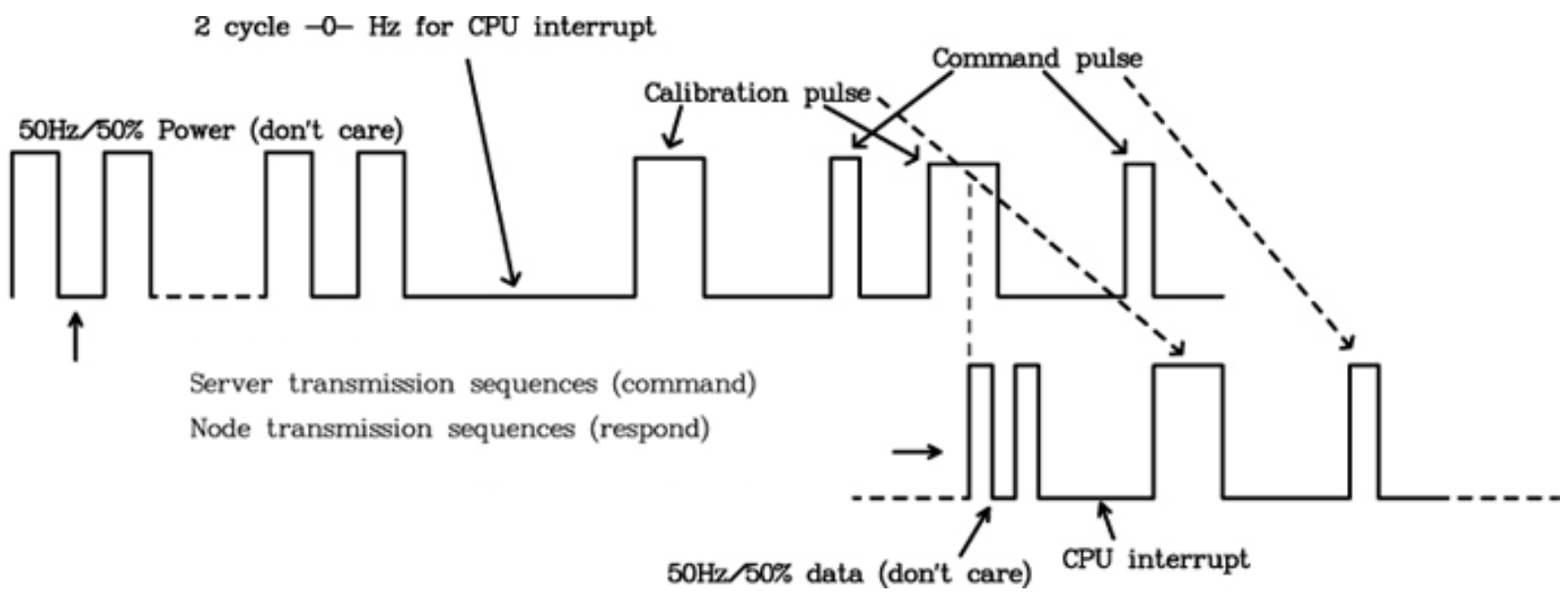

Figure 8. Calibration.

and 2 calculates the amount of count loss. Let's assume that the embedded CPU2 counter value has reached to 4897 within the interval of the receiving pulse. Therefore, $C_{\text {cal }}=103$ pulses are added to the next coming pulse. Figure 8 shows a sample of commander and data pulses accompanied by a calibration pulse in advance.

\subsection{Communication Protocol}

There are plenty of methods of communication using FAMPLC technique. The initial concept was based on an openly user-defined protocol called FAM protocol. However, conventional asynchronous serial transmission has been also experienced using this technique. Nevertheless, a method in which the Data Envelope Analysis (DAE) to measure the productive efficiency of a Decision Making Unit (DMU) can be considered [11]. In this project, as a preferred point to multipoint model, frequency is used to enable the desired node and duty cycle is to carry the information for that particular node. In this method the transmission from each node is made only with the permission or request from the server. To enhance the accuracy of the transmission, two or more informative pulses shall be transmitted from each side. If sensors and devices of all the nodes are alike, since each node's transmission is followed by a permission or request and server expects to receive data from the Permitted To Talk (PTT) node, predefined commander pulse widths can be considered equal for all the nodes. In this case, each receiver node is woken up with a dedicated frequency as an ID.

\section{Conclusion}

This paper is exclusively about the hardware architecture and a mathematical proposal for an optimum utilization of the FAMPLC technique to access multiple remote I/Os. The unique mathematical approach for frequency optimization in the presented point to multipoint model can be used for design of either an uncategorized or a categorized model. The offered calibration method has shown an effective compensation of the lost counts in harsh conditions. This novel technique works based on the user defined protocol and has yet to be improved and further study shall be conducted for an introduction of a unique communication protocol as the future work.

\section{Acknowledgement}

Thanks to the Universiti Sains Malaysia for facilitating this research by the PRGS 1001/PELECT/8043012 grant scheme which helped to motivate the approach for the research's outcomes.

\section{References}

1. Vaidya B, Park J H et al. (2010). Robust one-time password authentication scheme using smart card for home network environment, Computer Communications, vol 34(3), 326-336.

2. Skjetne R, Jørgensen U et al. (2011). Line-of-sight pathfollowing along regularly parametrized curves solved as a generic maneuvering problem, Proceedings of 2011 50th IEEE 
Conference on Decision and Control and European Control Conference (CDC-ECC), Orlando, FL, USA, 2467-2474.

3. Figueiredoa J, and Costab J S D (2012). A SCADA system for energy management in intelligent buildings, Energy and Buildings, vol 49, 85-98.

4. Kirubashankar R, Krishnamurthy K et al. (2009). Remote monitoring system for distributed control of industrial plant process, Journal of Scientific \& Industrial Research, vol 68(10), 858-860.

5. Park H, Song J et al. (2012). Comparative study on position of shunt active power filters in $25 \mathrm{KV}$ AC railway systems, IETE Technical Review, vol 29(5), 421-431.

6. Nazem A, and Arshad M R (2012). Real time distant communication using frequency and amplitude combined modulations in single source power line, Proceedings of International Conference on Advances in Electrical and Electronics Engineering, Malaysia, 330-335.
7. Nazem A, and Arshad M R (2012). Frequency modulated power supply used to navigate the Remotely Operated Vehicle (ROV), Proceedings of 4th International Conference on Intelligent and Advanced Systems, Malaysia, vol 1, 222-225.

8. Nazem A, and Arshad M R (2012). A dummy active resistance to transmit the navigational parameters of an ROV, Procedia Engineering, vol 41, 175-181.

9. Microchip (1997). PICmicro ${ }^{\mathrm{m}}$ mid-range MCU family reference manual, 1-1-1-5.

10. Karfa C (2012). Applications of behavioural transformations in embedded system design, IETE Technical Review, vol 29(5), 372-379.

11. Malkhalifeh M R, Mollaeian E et al. (2013). A new nonradial network DEA model for evaluating performance supply chain, Indian Journal of Science and Technology, vol 6(3), 4187-4192. 